\title{
A Clinical Analysis of Sudden Sensorineural Hearing Loss Cases
}

\author{
Hyun Soo Lee ${ }^{1}$, You Jae Lee ${ }^{1}$, Bo Sung Kang ${ }^{1}$, Byung Don Lee ${ }^{1}$, and Ji Sung Lee ${ }^{2}$ \\ ${ }^{1}$ Department of Otolaryngology-Head and Neck Surgery, Soonchunhyang University College of Medicine, Seoul, \\ ${ }^{2}$ Biostatistical Consulting Unit, Soonchunhyang University Medical Center, Seoul, Korea
}

Received April 17, 2014

Revised July 12, 2014

Accepted July 23, 2014

\section{Address for correspondence Byung Don Lee, MD, PhD \\ Department of Otolaryngology- \\ Head and Neck Surgery, \\ Soonchunhyang University \\ College of Medicine, \\ 59 Daesagwan-ro, Yongsan-gu, \\ Seoul 140-743, Korea \\ Tel +82-2-709-9364 \\ Fax +82-2-709-9134 \\ E-mail bdlee12@schmc.ac.kr}

Background and Objectives: High-dose systemic steroid therapy is the mainstay treatment for sudden sensorineural hearing loss (SSNHL). Recovery rates from SSNHL range are about $47-63 \%$ and are influenced by various prognostic factors. To evaluate the prognostic value of specific clinical parameters, we reviewed 289 cases by clinical and statistical analysis. Subjects and Methods: This study included 289 patients with SSNHL who visited the Department of Otolaryngology at Soonchunhyang University Hospital from January 2005 to December 2012. The cases were reviewed retrospectively based on clinical charts. Hearing improvement was evaluated in relation to pure-tone audiogram results, duration between SSNHL onset and time of initial treatment, seasonal incidence, dizziness, patient age, degree of hearing loss, patterns of initial pure-tone audiogram and presence of underlying disease. Results: Hearing improvement was observed in 196 of 289 (67.8\%) patients; such improvement began within 7 days in most patients, followed by rapid hearing recovery. Cases that failed to show improvement within 14 days were unlikely to achieve hearing recovery. The more severe the hearing loss during the early stage, the lower the hearing recovery rates. Patients aged less than 60 years appear to have better prognosis of hearing improvement compared to those who are over 60 years. Conclusions: Important prognostic factors for recovery in patients with SSNHL include the time of initiating treatment after symptom onset, the degree of early-stage hearing loss, and the age of the affected patient.

Korean J Audiol 2014;18(2):69-75

\section{Introduction}

Sudden sensorineural hearing loss (SSNHL) is an audiologic emergency disease characterized by sudden hearing loss that affects 5-20 per 100000 individuals annually. ${ }^{1)}$ SSNHL usually occurs unilaterally. Causes include infectious diseases, blood vessel disorders and autoimmune diseases; however, the etiology is ambiguous in most cases of SSNHL. Basic regimes for treating patients with SSNHL consist of rest, a lowsalt diet, and short-term high-dose steroid injections. Additional treatments for SSNHL include adrenal cortical hormone medicines, blood circulation improvement medicines, vasodilators, anti-viral drugs, diuretics, hyperbaric oxygen therapy, and stellate ganglion blocks. ${ }^{2-5)}$ The natural recovery rate of SSNHL is $47-63 \%$ and in most cases, recovery occurs within 2

This is an Open Access article distributed under the terms of the Creative Commons Attribution Non-Commercial License (http://creativecommons. org/licenses/by-nc/3.0/) which permits unrestricted non-commercial use, distribution, and reproduction in any medium, provided the original work is properly cited. weeks. ${ }^{6}$

Various prognostic factors have been evaluated for their capacity to predict recovery from SSNHL including age, dizziness, degree of early-stage hearing loss, type of hearing loss, time of initiating treatment, and systemic diseases such as diabetes mellitus and hypertension. ${ }^{5,78)}$ However, little is known about the temporal relationship between the clinical course of patients with SSNHL and their hearing recovery; the timing and duration of hearing recovery remain unclear. Moreover, the prognostic factors that affect the recovery rate have not been sufficiently described. Thus, we analyzed the associations between prognostic factors and successful treatment of SSNHL and recovery in affected patients.

\section{Subjects and Methods}

A total of 289 patients diagnosed with SSNHL at the Seoul and Gumi hospitals of Soonchunhyang University from January 1,2005, to December 31, 2012, were included. All patients 
received the same treatment during hospitalization and received appropriate follow-up care post-hospitalization. All patients underwent physical examination of the eardrum and cranial nerves, hearing ability tests (including the auditory brainstem response test), temporal bone magnetic resonance imaging, fistula tests, and vestibular function tests. Cases with inflammation in the middle or inner ear were excluded. Hearing tests were conducted using the following schedule; once at the time of admission, once every other day during the time of hospitalization after the treatment began, once a week for the first month after being discharged from the hospital, and once a month thereafter. The scheduled hearing tests were conducted over a 12 month period in patients with slight hearing recovery and in non-respondents.

The diagnostic criterion for SSNHL was more than 30 decibel hearing loss (dB HL) in three consecutive frequencies within 3 days of symptom onset. All patients diagnosed with SSNHL received absolute rest and low-salt diet for 7 days. Medical therapy included $10 \mathrm{mg}$ dexamethasone injection for 5 days, which was reduced to $7.5 \mathrm{mg}$ on Days 6 and 7. Upon discharge, steroid therapy was converted into $20 \mathrm{mg}$ oral prednisolone (Solondo, $5 \mathrm{mg} /$ tablet, Yuhan Corp., Seoul, Korea) on Days 8 and 9, which was then reduced to $10 \mathrm{mg}$ on Day 10. In addition, the vasodilator Gingko flavone glycoside (Tanamin, 80 mg/tablet, Yuyu Pharma, Inc., Seoul, Korea) was administered as a supplement, and a stellate ganglion block was performed.

The following prognostic factors were selected for analysis: age, systemic diseases (e.g., hypertension and diabetes mellitus), dizziness, degree of early-stage hearing loss, type of hearing loss, and time of initiating treatment. The relationships between hearing recovery rate and these prognostic factors were analyzed.

The degree of hearing loss was measured using the average threshold value (dB HL), which was derived from the method of quartering of $0.5,1,2$, and $3 \mathrm{kHz}$ using pure-tone audiometry. ${ }^{9)}$ Hearing loss was classified as mild (26-40 dB), moderate $(41-55 \mathrm{~dB})$, moderately severe $(56-70 \mathrm{~dB})$, severe (71$90 \mathrm{~dB}$ ), or profound ( $\geq 91 \mathrm{~dB}){ }^{9)}$ The hearing threshold value was used as a criterion according to Shaia and Sheehy. ${ }^{3)}$ Thus, the pure-tone audiograms were classified as follows: rising (lower threshold values in the high-frequency range of 2000$4000 \mathrm{~Hz}$ than in the low-frequency range of 250-500 Hz), flat (similar threshold observed across the entire frequency range), and sloping (higher threshold values in the high-frequency range than in the low-frequency range). ${ }^{3,10)}$ Cases were classified as profound when hearing loss was profound and classification was not possible.

Patients degree of recovery was classified into four groups according to Siegel's method of classification; ${ }^{11)}$ complete recovery, partial recovery, slight recovery, and non-recovery. The complete recovery group included patients with final hearing abilities $<25 \mathrm{~dB}$ HL. The partial recovery group included patients with hearing recovery $>15 \mathrm{~dB}$ HL with final hearing abilities between $25-45 \mathrm{~dB}$ HL. The slight recovery group included patients with hearing recovery of $>15 \mathrm{~dB}$ HL with final hearing abilities $>45 \mathrm{~dB}$ HL. The non-recovery group included patients with hearing recovery $<15 \mathrm{~dB}$ HL with final hearing abilities $>75 \mathrm{~dB}$ HL.

The chi-squared test and logistic regression analyses were conducted using SPSS for Windows 12.0 (SPSS Inc., Chicago, IL, USA) and the chi-squared test for one-way analysis using SAS 9.3 (SAS Institute, Cary, NC, USA). Results were considered significant when the $p$-value was below 0.05 .

\section{Results}

\section{Incidence rate of SSNHL by sex, age, and season}

Among the 289 patients who received treatments for SSNHL, 161 were female $(55.7 \%)$ and 128 were male $(44.3 \%)$ (Table 1 ). The average age of affected patients was 50.8 (range, 977) years; the average age in females was 50.6 years, and the average age males was 51.1 years. The incidence rate of SSNHL was highest (45.0\%) among subjects in their $50 \mathrm{~s}$ and $60 \mathrm{~s}(130$ individuals). Seasonally, SSNHL occurred more often in December and January than between June and August; however, this trend was not statistically significant.

\section{Hearing recovery rate}

The distribution of patients (Fig. 1) according to the recovery of hearing ability based on the Siegel classification ${ }^{11)}$ was as follows: $37.0 \%(n=107)$ achieved complete recovery, $16.6 \%$ $(\mathrm{n}=48)$ showed partial recovery, $14.2 \%(\mathrm{n}=41)$ showed slight recovery, and $32.2 \%(n=93)$ showed non-recovery. The overall recovery rate including the slight recovery group was $67.8 \%$.

\section{Hearing recovery period}

The average hearing recovery period was 7.1 days for complete recovery, 13.2 days for partial recovery, and 24.1 days for slight recovery. No further hearing recovery was observed beyond 60 days.

\section{Onset of hearing recovery after the treatment}

In cases with complete hearing recovery, rapid recovery began on Day 3, and normal hearing returned by Day 7. In cases with partial hearing recovery, the recovery started on Day 3, but unlike the cases with complete recovery, the rapid recovery phase started on Day 7. In cases with slight hearing recov- 
Table 1. Hearing improvement of patients with sudden sensorineural hearing loss in relation to the prognostic factors

\begin{tabular}{|c|c|c|c|c|}
\hline Prognostic indicator & No. of patients & R group (\%) & N group (\%) & $p$-value \\
\hline Age distribution range & & & & $<0.001$ \\
\hline Under 60 & 184 & $140(71.4)$ & $44(47.3)$ & \\
\hline Over 60 & 105 & $56(28.6)$ & $49(52.7)$ & \\
\hline Sex & & & & 0.579 \\
\hline Male & 128 & $89(45.4)$ & $39(41.9)$ & \\
\hline Female & 161 & $107(54.6)$ & $54(58.1)$ & \\
\hline \multicolumn{5}{|l|}{ Associated conditions } \\
\hline Diabetes mellitus & 48 & $29(31.2)$ & $19(32.8)$ & 0.229 \\
\hline Chronic renal failure & 9 & $6(6.5)$ & $3(5.2)$ & 1.000 \\
\hline Hypertension & 48 & $31(33.3)$ & $17(29.3)$ & 0.599 \\
\hline Dizziness & 46 & $27(29)$ & $19(32.8)$ & 0.149 \\
\hline Hearing level & & & & $<0.001$ \\
\hline Mild, $26-40 \mathrm{~dB}$ & 39 & $36(18.4)$ & $3(3.2)$ & \\
\hline Moderate, $41-55 \mathrm{~dB}$ & 57 & $38(19.4)$ & $19(20.4)$ & \\
\hline Moderate-severe, 56-70 dB & 73 & $46(23.5)$ & $27(29)$ & \\
\hline Severe, $71-90 \mathrm{~dB}$ & 66 & $53(27.0)$ & $13(14.0)$ & \\
\hline Profound, over $91 \mathrm{~dB}$ & 54 & $23(11.7)$ & $31(33.3)$ & \\
\hline Audiogram types & & & & $<0.001$ \\
\hline Sloping & 57 & $41(21)$ & $16(17.2)$ & \\
\hline Flat & 151 & $104(53.1)$ & $47(50.5)$ & \\
\hline Rising & 56 & $48(24.5)$ & $8(8.6)$ & \\
\hline Profound & 25 & $3(1.5)$ & $22(23.7)$ & \\
\hline Duration from onset to treatment (days) & & & & $<0.001$ \\
\hline Within 7 day & 242 & $179(91.3)$ & $63(67.7)$ & \\
\hline Over 8 day & 47 & $17(8.7)$ & $30(32.3)$ & \\
\hline
\end{tabular}

R group: complete, partial, slight recovery group, $\mathrm{N}$ group: no response, $\mathrm{p}$-value: chi-square test

ery, the recovery started on Day 5, which was gradual, and continued until Day 14. Little change in hearing was observed after 28 days; no further hearing improvement was observed after 60 days. Patients who did not respond to the treatment after 2 weeks of treatment failed to show complete hearing recovery. However, two patients showed partial hearing recovery, and four showed slight hearing recovery (Fig. 2).

\section{Relationships between prognostic factors and recovery rate}

Age

Patients $<60$ years of age showed a high hearing recovery rate of $71.4 \%$. This was significantly greater than that in subjects $\geq 60$ years of age, who showed a hearing recovery rate of $28.6 \%$ (Fig. 3, Table 1). No significant difference was detected in patients $<60$ years who were divided into two groups $(<45$ vs. $\geq 46$ years of age; $p=0.610)$.

\section{Systemic diseases}

A total of $48(16.6 \%), 48(16.6 \%)$, and $9(2.4 \%)$ patients had hypertension, diabetes mellitus, or chronic renal failure, respectively, with no significant differences among groups ( $p>$

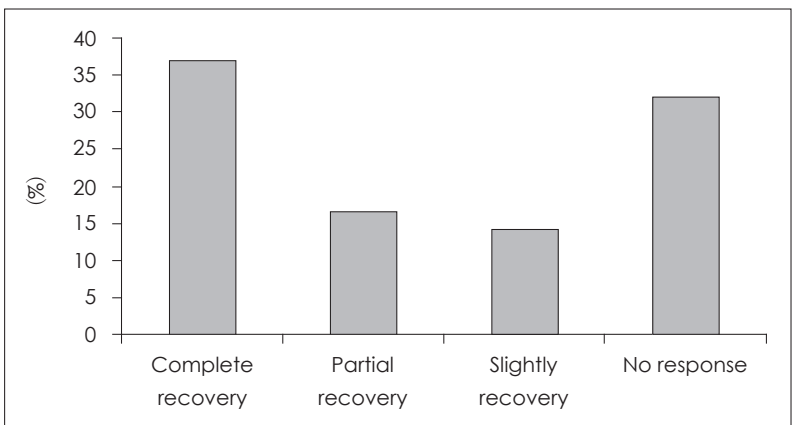

Fig. 1. Hearing improvement of sudden sensorineural hearing loss $(n=289)$.

\section{$0.05)($ Table 1).}

\section{Dizziness}

In 46 cases $(15.9 \%)$ in which vertigo was present, the hearing recovery rate was $29 \%$, which was not significantly different from that of the patients without vertigo $(p=0.14)$. The vestibular function test was not performed routinely (Table 1).

\section{Degree of hearing loss in the early stage}

The recovery rate depended on the degree of hearing loss at the first visit. Recovery rates were $94.1 \%$ in patients with mild 

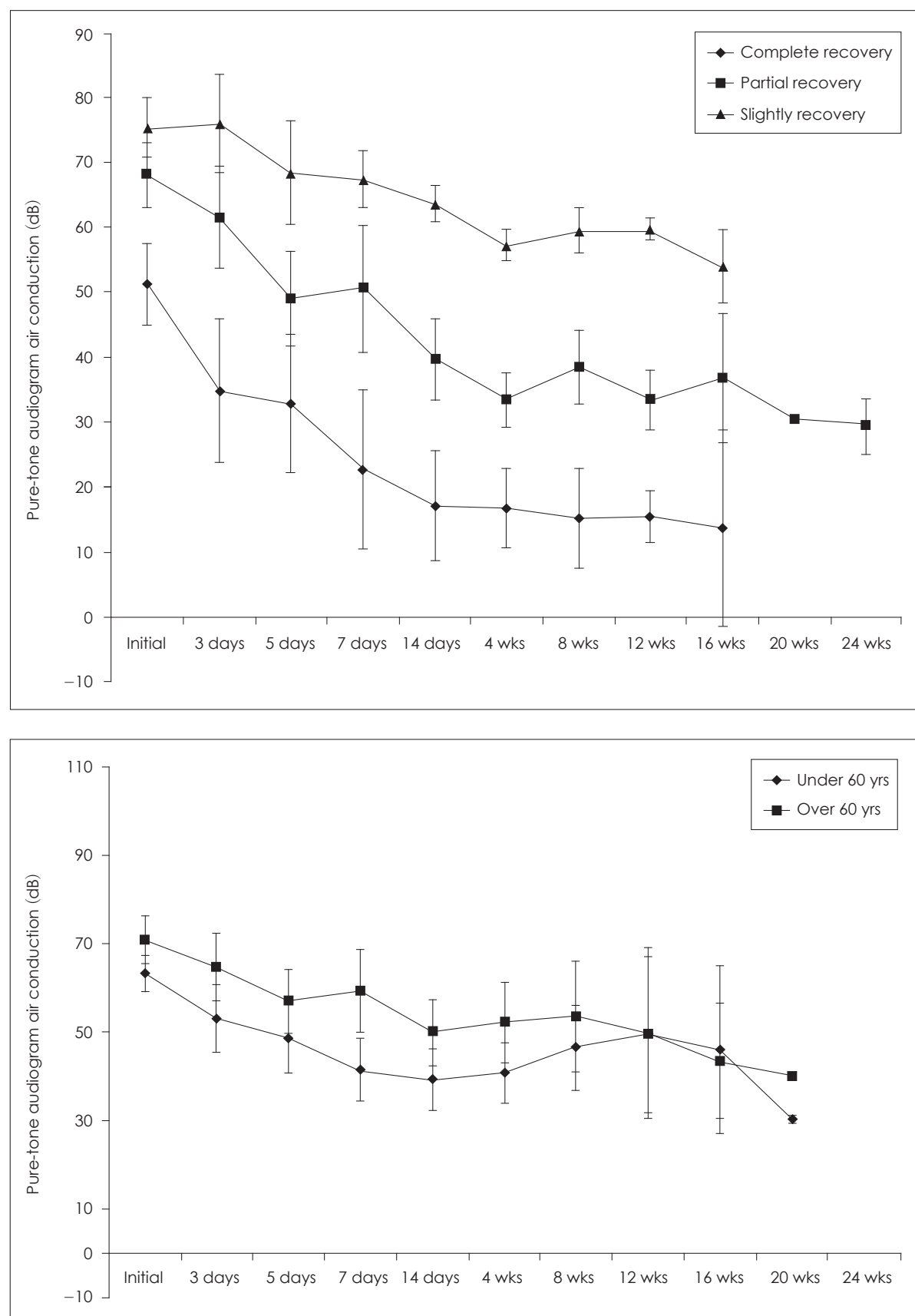

Fig. 2. Time-dependent patterns of hearing recovery in patients from the three recovery groups. As the final hearing status was poorer, the speed of recovery seems to be delayed. The bar means $95 \%$ confidence interval of the mean. wk(s): week(s).
Fig. 3. Time-dependent patterns of hearing recovery in patients from two age group. The bar means $95 \%$ confidence interval of the mean. wk(s): week(s). hearing loss, $90.0 \%$ in patients with moderate hearing loss, $61.9 \%$ in patients with moderately severe hearing loss, $80.0 \%$ in patients with severe hearing loss, and $42.6 \%$ in patients with profound hearing loss. The more severe the hearing loss during the early stage, the lower the hearing recovery rates. In addition, the final clinical outcome showed only slight or partial recovery rather than complete recovery $\left(\chi^{2}\right.$ test for linear trend $p=0.001$ )(Fig. 4).

\section{Patterns of initial pure-tone audiogram}

Regarding the pure-tone audiograms, $19.7 \%$ of affected pa- tients displayed sloping audiogram patterns, 52.2\% had flat patterns, $19.4 \%$ had rising patterns, and $8.7 \%$ displayed profound hearing loss. Unlike other studies that have reported sloping or rising patterns as the most common types, the most common pattern in this study was the flat audiogram. This discrepancy could be explained by the fact that we divided the audiogram patterns into only four categories (i.e., sloping, flat, rising, and profound), whereas most previous studies have included six categories, dividing the flat type in greater detail. ${ }^{8)}$ The hearing recovery rates were investigated by analyzing initial audiogram patterns. Hearing recovery was observed in 

hearing recovery in patients from the severity the hearing loss groups during the early stage. The bar means $95 \%$ confidence interval of the mean. wk(s): week(s), HL: hearing loss.
Fig. 4. Time-dependent patterns of

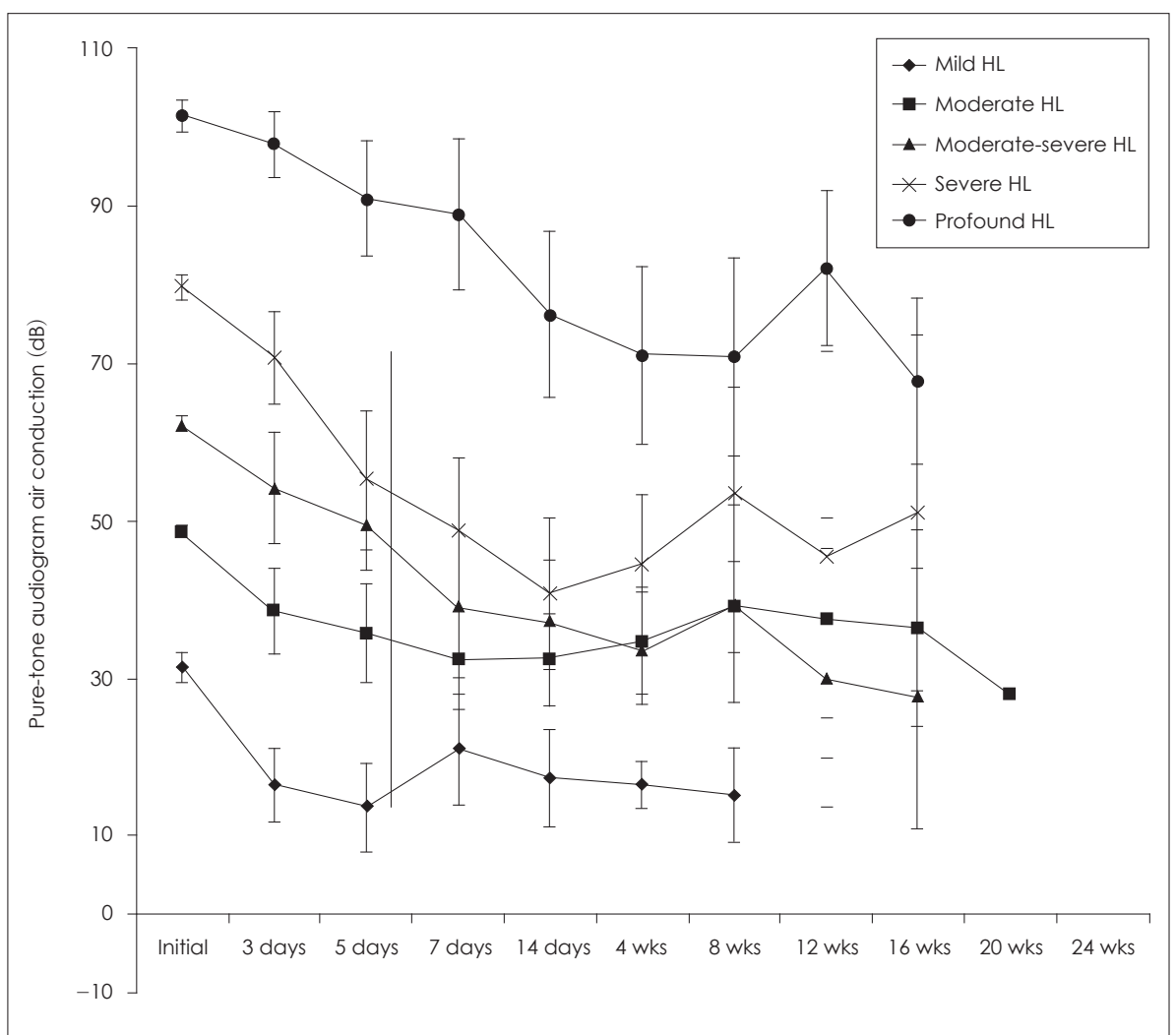

$68.8 \%$ of subjects displaying the flat type, $71.9 \%$ of subjects showing the rising type, and $85.7 \%$ of subjects displaying the sloping type. Three subjects showed higher than profound type of $12.0 \%$. This was statistically significant $(p=0.003)$ (Fig. 5).

\section{Starting time of treatment}

The recovery rate was $73.8 \%$ in subjects who received their initial treatment within 3 days, $87.2 \%$ within 7 days, and $<30 \%$ after 7 days. The recovery rate was significantly higher in cases that received initial treatment within 7 days from the onset of the disease compared to those receiving initial treatment after 7 days $(p<0.001)$. However, no significant difference was detected between groups receiving initial treatment within 3 days and those receiving initial treatment within 7 days $(p=0.968)$.

\section{Characteristics of the non-recovery group}

A total of 93 patients showed no hearing recovery. Of these, 31 had profound hearing loss in the early stage, and 33.3\% of these patients had no response to recovery. This study included 47 patients who received their initial treatments after 7 days, 30 of whom (32.3\%) belonged to this non-recovery group. Furthermore, 49 patients were over 60 years of age, accounting for a significant proportion $(52.7 \% ; p<0.001)$ of the non-

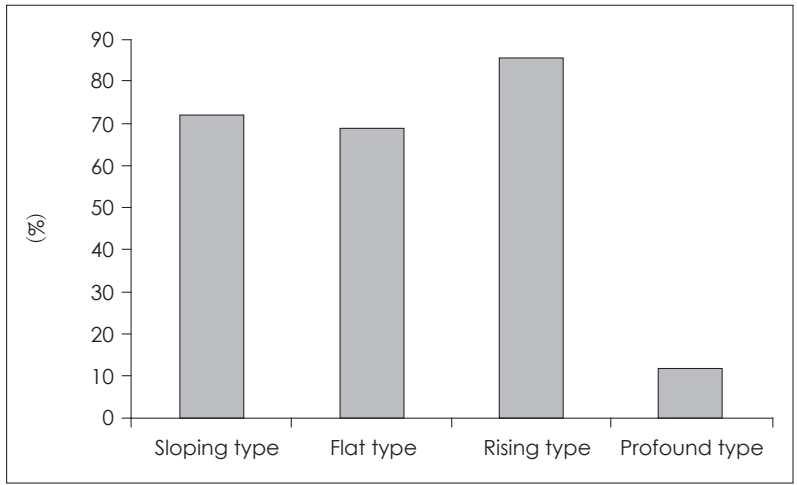

Fig. 5. Recovery rate by patterns of initial pure tone audiogram.

recovery group (Table 1 ).

The degree of recovery did not differ between subjects with and without a medical condition. This was not statistically significant $(p=0.34)$. A total of 58 patients had a medical history of hypertension, diabetes mellitus, chronic renal failure, or dizziness; 18 subjects had two of the four conditions, 8 had three of the four conditions, and 2 had all four medical conditions.

\section{Discussion}

SSNHL is a sensorineural hearing loss that occurs suddenly within hours or days with no clear cause. SSNHL is diagnosed 
in patients with a sensorineural hearing loss of $>30 \mathrm{~dB}$ in three or more consecutive frequencies occurring within 3 days. Causes of SSNHL include viral infection, blood vessel disorder, inner ear membrane rupture, and autoimmune disease. ${ }^{12)}$ SSNHL is managed using a variety of treatments including high-concentration steroid injection for anti-inflammation or vasodilation. ${ }^{2,5,6)}$ Steroid treatments restrain the inflammatory reaction, activate ion transport in the stria vascularis and spiral ligament within the cochlear duct, control homeostasis of the endolymph, and increase blood circulation in the cochlea. ${ }^{13-15)}$

SSNHL affects both sexes equally; it can occur across a wide age range, but it occurs most often in individuals in their $30 \mathrm{~s}$ to $50 \mathrm{~s}$. The condition is commonly unilateral, but it is bilateral in less than $1.7 \%$ of patients. ${ }^{16)}$ In this study, females were affected more than males, and most cases occurred in patients in their $50 \mathrm{~s}$ to $60 \mathrm{~s}$, but There were no significant differences between the sexes. No bilateral cases of SSNHL were observed. The incidence rate of SSNHL was higher in December $(12.1 \%)$ and January $(11.4 \%)$, but these rates were not significantly different from those in other months $(p=0.289)$.

This study investigated the efficacy of treatment for SSNHL. However, it was difficult to establish a control group and to independently assess the various factors affecting prognosis. In addition, this study was further limited because various medicines were administered together with steroids in many cases. Therefore, stringent guidelines were applied to reduce the number of parameters in this study. Only patients diagnosed with SSNHL, who received hospital treatment for 7 days and were observed for a minimum of 1 month as outpatients, were chosen as subjects for this study.

In general, even without treatment, spontaneous recovery from SSNHL can occur within 2 weeks. Moreover, 47-63\% ${ }^{7)}$ of affected patients show at least slight recovery. ${ }^{7,17)}$ In the present study, the overall recovery rate including slight recovery was $67.8 \%$, which is consistent with other studies. ${ }^{7,17)}$

The hearing recovery period is within $2-4$ weeks in most cases. ${ }^{7)}$

In the present study, complete recovery was achieved in an average of 7.1 days, and 155 patients (53.6\%) showed more than partial recovery within an average of 13.2 days. Moreover, 144 patients $(66.4 \%)$ showed more than slight recovery within an average of 24.1 days. These outcomes are consistent with those reported in other studies.

In most cases in the present study, recovery began on Day 3 of treatment followed by rapid recovery within 7 days. Thereafter, recovery proceeded slowly with little change in hearing improvement after 24 days. It was not possible to determine whether the continued gradual hearing improvement beyond 24 days from the start of treatment was due to the treatment or the natural healing process. It is notable that gradual hearing recovery continued for 2 months after completion of treatment in some cases. However, no further recovery was observed beyond the initial 2 month period.

This critical recovery period suggests that hearing recovery requires hospital treatment and outpatient care for 7-14 days. Patient recovery should be monitored on an outpatient basis for a minimum of 2 months. Existing treatment options, additional medication types, patient education, and administration methods such as intratympanic steroid injection require further development. It is important to establish proper treatment principles to provide the best index not only for patients but also for clinicians.

Hearing recovery rates are influenced by various prognostic factors such as age, systemic diseases (e.g., hypertension and diabetes mellitus), and other factors as mentioned above. ${ }^{7,18)}$

We analyzed six prognostic factors with regard to hearing recovery rate. The recovery rate was significantly higher in patients $<60$ years of age $(71.4 \%)$, which was higher than the natural recovery rate. ${ }^{6}$ The recovery rate in patients $\geq 60$ years was $28.6 \%$, which was lower than the natural recovery rate. As shown in Fig. 3, the hearing abilities in patients $<60$ years seemed to decline over time. This could be due to the small number of patients with longer follow-up care, which consisted of a group of patients with no hearing recovery. Hospital treatment is recommended; additional treatment plans for hearing recovery need to be established for patients aged $\geq 60$ years. Cases with accompanying systemic diseases such as hypertension and diabetes mellitus showed recovery rates of $63 \%$. This rate is higher than the $49.5 \%$ recovery rate in another report, ${ }^{8)}$ but not statistically significantly different. Nevertheless, a comprehensive and focused approach involving cooperative medical treatment in other departments may help patients cope with diseases and hearing loss.

The recovery rate in cases with accompanying dizziness was $58.6 \%$, higher than the $47.8 \%$ recovery rate reported elsewhere. ${ }^{19)}$ This difference could be due to the fact that we included all subjective symptoms of dizziness, in addition to the objective findings of nystagmus or decreased vestibular function. Therefore, it is important to consider the affected ear and degree of dizziness, in conjunction with conducting a vestibular function test.

A previous study reported a $30 \%$ recovery rate in patients with severe (and greater) initial hearing loss, and an $86.3 \%$ recovery rate in patients with moderately severe (and less) initial hearing loss. ${ }^{8)}$ We found similar results: $42.9 \%$ in patients with severe initial hearing loss, and $83.0 \%$ in patients with moderately severe initial hearing loss. This suggests that recovery rate drops as the severity of initial hearing loss increas- 
Table 2. Hearing improvement of patients with sudden sensorineural hearing loss in relation to the prognostic factors

\begin{tabular}{|c|c|c|c|c|}
\hline \multirow{2}{*}{ Prognostic indicator } & \multirow[t]{2}{*}{ OR } & \multicolumn{2}{|c|}{$\begin{array}{l}95 \% \text { confidence } \\
\text { interval }\end{array}$} & \multirow{2}{*}{$p$-value } \\
\hline & & Lower & Upper & \\
\hline \multicolumn{5}{|l|}{ Age } \\
\hline Under 60 & Ref & & & \\
\hline Over 60 & 0.259 & 0.137 & 0.489 & $<0.001$ \\
\hline \multicolumn{5}{|l|}{ Hearing level } \\
\hline Mild & Ref & & & \\
\hline Moderate & 0.221 & 0.055 & 0.882 & 0.033 \\
\hline Moderate-severe & 0.177 & 0.046 & 0.684 & 0.012 \\
\hline Severe & 0.386 & 0.094 & 1.580 & 0.185 \\
\hline Profound & 0.221 & 0.047 & 1.029 & 0.054 \\
\hline \multicolumn{5}{|l|}{ Audiogram types } \\
\hline Sloping & Ref & & & \\
\hline Flat & 0.792 & 0.375 & 1.674 & 0.542 \\
\hline Rising & 1.665 & 0.578 & 4.795 & 0.345 \\
\hline Profound & 0.035 & 0.007 & 0.178 & $<0.001$ \\
\hline \multicolumn{5}{|l|}{ Onset to treatment } \\
\hline Within 7 day & Ref & & & \\
\hline Over 8 day & 0.136 & 0.063 & 0.295 & $<0.001$ \\
\hline
\end{tabular}

p-value: logistic regression analysis. OR: odds ratio

es. The hearing abilities of some patients worsened over time (Fig. 4).

In contrast to a previous study, ${ }^{6,18)}$ we detected no significant differences in the recovery rates among groups based on patterns of initial pure-tone audiograms. When we performed logistic regression analysis using the sloping type from Table 2 as the reference, we could not find statistical usefulness on other types.

We analyzed recovery rate with respect to the time treatment was initiated. A total of 122 subjects $(56.2 \%)$ presented to our hospital within 3 days of occurrence. In contrast to other studies that used 7 day intervals, we analyzed intervals that were divided into two groups: $1-3$ days and 4-7 days after the onset of SSNHL. No significant difference was detected between the two groups in terms of hearing recovery rate. However, the rate was significantly lower in cases treated 7 days after disease onset compared to those treated within 7 days, consistent with another report. ${ }^{5)}$ Many patients who failed to recover their sense of hearing received initial treatment 7 days after symptom onset.

\section{Conclusion}

Important prognostic factors for recovery in patients with
SSNHL include the time of initiating treatment after symptom onset, the degree of early-stage hearing loss, and the age of the affected patient.

\section{REFERENCES}

1) Byl FM Jr. Sudden hearing loss: eight years' experience and suggested prognostic table. Laryngoscope 1984;94(5 Pt 1):647-61.

2) Haberkamp TJ, Tanyeri HM. Management of idiopathic sudden sensorineural hearing loss. Am J Otol 1999;20:587-92; discussion 593-5.

3) Shaia FT, Sheehy JL. Sudden sensori-neural hearing impairment: a report of 1,220 cases. Laryngoscope 1976;86:389-98.

4) Park KH, Lee CK, Lee JD, Park MK, Lee BD. Combination therapy with systemic steroids, an antiviral agent, anticoagulants, and stellate ganglion block for treatment of sudden sensorineural hearing loss. Korean J Audiol 2012;16:71-4.

5) Choung YH, Lee SJ, Lee JW, Mo JY, Moon SK, Park K. The Comparison of Hearing Recovery of Sudden Deafness Treated with Different Protocols. Korean J Audiol 2003;7:24-31.

6) Mattox DE, Simmons FB. Natural history of sudden sensorineural hearing loss. Ann Otol Rhinol Laryngol 1977;86(4 Pt 1):463-80.

7) Lee MY, Suh MW, Rhee CK, Jung JY. Clinical characteristics of sudden sensorineural hearing loss with aggravation during treatment. Korean J Otorhinolaryngol-Head Neck Surg 2008;51:973-8.

8) Tiong TS. Prognostic indicators of management of sudden sensorineural hearing loss in an Asian hospital. Singapore Med J 2007;48: 45-9.

9) Melnick W. American National Standard specifications for audiometers. ASHA 1971;13:203-6.

10) Fikret K, Gokhan T, Ibrahim H, Levent E, Oguz B, Selcuk O, et al. Prognosis in sudden hearing loss: is it the disease or the treatment that determines the prognosis? J Int Adv Otol 2009;5:187-94.

11) Siegel LG. The treatment of idiopathic sudden sensorineural hearing loss. Otolaryngol Clin North Am 1975;8:467-73.

12) Wilson WR, Veltri RW, Laird N, Sprinkle PM. Viral and epidemiologic studies of idiopathic sudden hearing loss. Otolaryngol Head Neck Surg 1983;91:653-8.

13) Erichsen S, Bagger-Sjöbäck D, Curtis L, Zuo J, Rarey K, Hultcrantz M. Appearance of glucocorticoid receptors in the inner ear of the mouse during development. Acta Otolaryngol 1996;116:721-5.

14) Erichsen S, Zuo J, Curtis L, Rarey K, Hultcrantz M. Na, K-ATPase alpha- and beta-isoforms in the developing cochlea of the mouse. Hear Res 1996;100:143-9.

15) Trune DR, Wobig RJ, Kempton JB, Hefeneider SH. Steroid treatment improves cochlear function in the MRL.MpJ-Fas(lpr) autoimmune mouse. Hear Res 1999;137:160-6.

16) Fetterman BL, Saunders JE, Luxford WM. Prognosis and treatment of sudden sensorineural hearing loss. Am J Otol 1996;17:529-36.

17) Yoon YJ, Kim BK, Lim HS, Choi SC. Clinical analysis of sudden hearing loss. Korean J Audiol 2001;5:83-90.

18) Shin SO. Clinical characteristics and management of sudden sensorineural hearing loss. Korean J Audiol 2011;15:1-7.

19) Lee DH, Yeo SW, Park JO, Shim MB, Choi JY. Clinical analysis of sudden sensorineural hearing loss; focused on underlying diseases as risk factor. Korean J Audiol 2004;8:58-65. 